\title{
Engineering of $\mathrm{CHO}$ cells for the production of vertebrate recombinant sialyltransferases
}

\author{
Benoit Houeix ${ }^{1}{ }^{\text {, Michael T Cairns }}{ }^{\text {Corresp. } 1}$ \\ ${ }^{1}$ Glycoscience Group, National Centre for Biomedical Engineering Science, National University of Ireland, Galway, Galway, Ireland \\ Corresponding Author: Michael T Cairns \\ Email address: michael.cairns@nuigalway.ie
}

Background. Sialyltransferases are a family of enzymes that transfer sialic acid to glycan chains on glycoproteins, glycolipids and oligosaccharides. They play key roles in determining cell-cell and cell-matrix interactions and are important in neuronal development, immune regulation, protein stability and clearance. Most fully characterised sialyltransferases are of mammalian origin and these have been used for in vitro and in vivo modification of glycans. Additional versatility could be achieved by the use of animal sialyltransferases from other species that live in much more variable environments. Our aim was to generate a panel of stable $\mathrm{CHO}$ cell lines expressing a range of vertebrate sialyltransferases with different physicochemical and functional properties. Methods. The soluble forms of various animal ST6Gal and ST3Gal enzymes were stably expressed from a Gateway-modified secretion vector in $\mathrm{CHO}$ cells. The secreted proteins were IMAC-purified from serum-free media. Functionality of the protein was initially assessed by lectin binding to the host $\mathrm{CHO}$ cells. Activity of purified proteins was determined by a number of approaches that included a phosphate-linked sialyltransferase assay, HILIC-HPLC identification of sialyllactose products and enzyme-linked lectin assay (ELLA). Results. A range of sialyltransferase from mammals, birds and fish were stably expressed in CHO FlpIn cells. The stable cell lines expressing ST6Gal1 modify the glycans on the surface of the $\mathrm{CHO}$ cells as detected by fluorescently labelled lectin microscopy. The catalytic domains, as isolated by Ni Sepharose from culture media, have enzymatic activities comparable to commercial enzymes. Sialyllactoses were identified by HILIC-HPLC on incubation of the enzymes from lactose or whey permeate. The enzymes also increased SNA-I labelling of asialofetuin when incubated in a plate format. Conclusion. Stable cell lines are available that may provide options for the in vivo sialylation of glycoproteins. Proteins are active and should display a variety of biological and physicochemical properties based on the animal source of the enzyme. 
1 Engineering of $\mathrm{CHO}$ cells for the production of vertebrate recombinant sialyltransferases

2 Benoit Houeix $^{1,2}$ and Michael T Cairns ${ }^{1}$

3

$4 \quad{ }^{1}$ Glycoscience Group, National Centre for Biomedical Engineering Science, National University

5 of Ireland Galway, Galway, Ireland.

6

7 Corresponding author:

8 Michael Cairns

9 Email address: michael.cairns@nuigalway.ie

10

11

${ }^{2}$ Current address: Enterprise Ireland Medical and Engineering Technologies Gateway, Galway-

12 Mayo Institute of Technology, Galway, Ireland

13

14

15

16 


\section{ABSTRACT}

Background. Sialyltransferases are a family of enzymes that transfer sialic acid to glycan chains on glycoproteins, glycolipids and oligosaccharides. They play key roles in determining cell-cell and cell-matrix interactions and are important in neuronal development, immune regulation, protein stability and clearance. Most fully characterised sialyltransferases are of mammalian origin and these have been used for in vitro and in vivo modification of glycans. Additional versatility could be achieved by the use of animal sialyltransferases from other species that live in much more variable environments. Our aim was to generate a panel of stable $\mathrm{CHO}$ cell lines expressing a range of vertebrate sialyltransferases with different physicochemical and functional properties.

Methods. The soluble forms of various animal ST6Gal and ST3Gal enzymes were stably expressed from a Gateway-modified secretion vector in $\mathrm{CHO}$ cells. The secreted proteins were IMAC-purified from serum-free media. Functionality of the protein was initially assessed by lectin binding to the host $\mathrm{CHO}$ cells. Activity of purified proteins was determined by a number of approaches that included a phosphate-linked sialyltransferase assay, HILIC-HPLC identification of sialyllactose products and enzyme-linked lectin assay (ELLA).

Results. A range of sialyltransferase from mammals, birds and fish were stably expressed in FlpIn- $\mathrm{CHO}$ cells. The stable cell lines expressing ST6Gall modify the glycans on the surface of the $\mathrm{CHO}$ cells as detected by fluorescently labelled lectin microscopy. The catalytic domains, as isolated by Ni Sepharose from culture media, have enzymatic activities comparable to commercial enzymes. Sialyllactoses were identified by HILIC-HPLC on incubation of the enzymes from lactose or whey permeate. The enzymes also increased SNA-I labelling of asialofetuin when incubated in a plate format. 
40 Conclusion. Stable cell lines are available that may provide options for the in vivo sialylation of

41 glycoproteins. Proteins are active and should display a variety of biological and physicochemical

42 properties based on the animal source of the enzyme.

43

44

\section{INTRODUCTION}

Sialyltransferases (SIATs) transfer sialic acid (Sia) to glycan chains on glycoproteins and glycolipids. These sialylated structures are terminal structures on external cell surfaces therefore they are key players in cell-cell, cell pathogen and cell-matrix interactions, and are important factors in protein stability and clearance (Bragonzi et al. 2000; Choi et al. 2015; Olufsen et al. 2007), neuronal development (Galuska et al. 2006) and immune regulation (Hennet et al. 1998; Varki 1999). Some SIATs can also modify lactose to produce the sialyllactoses, 3 ' sialyllactose and $6^{\prime}$ sialyllactose, which are present in the new-born milk of many mammals. Human milk contains $12-14 \mathrm{~g} / \mathrm{L}$ milk oligosaccharides and is particularly rich in sialyllactoses $(\sim 20 \%)$. Evidence suggests that sialylated oligosaccharides possess anti-adhesive effects against certain pathogens and that sialyllactoses may increase adhesion of beneficial bacterial strains to the human intestine (Kavanaugh et al. 2013; Lane et al. 2012; Mysore et al. 1999). Although the sialyation of glycolipids and glycoproteins is universal to most tissues, the sialylation of lactose only occurs in mammary tissue. This process has been the subject of much interest because bovine milk, the source of most infant milk formula (IMF), is much lower in sialyllactoses than human milk (McJarrow \& van Amelsfort-Schoonbeek 2004). Producers of IMF are therefore developing processes to supplement bovine IMF with manufactured sialyllactoses in order to better mirror human milk. Dominant amongst these processes are so called 'one-pot' bacterial reaction systems (Fierfort \& Samain 2008). These are high yield and 
63 cost efficient systems where bacteria metabolise cheap simple sugars to produce sialyllactose.

64 All metabolic pathways within the bacteria that divert from the main pathway are knocked out

65

66

67

and novel SIAT genes, not naturally present in many bacterial species, are introduced for the final transfer of Sia to lactose. There are some causes for concern though. First of all, these introduced SIAT genes are bacterial, not mammalian: they carry out the same basic reaction but their protein sequences are only homologous to vertebrate proteins in some short sequence motifs (Meng et al. 2013). Bacterial SIATs are present only in select groups of bacteria (CAZy families GT30, GT38, GT42, GT73 and GT80) (Yamamoto et al. 2006) and many of these strains are highly pathogenic. The natural acceptor structures on bacterial strains are not the same structures as those recognised by vertebrate genes (as their natural role is to add Sia onto bacterial glycoproteins thereby mimicking vertebrate glycans). It is known that some of these SIATs have additional related activities and that they are much more flexible in their choice of acceptor and donor. In situations where, for example, the enzyme is required to add a novel modified sugar to a glycan, this is a distinct advantage, however, in the case of a food, it is essential to maintain high specificity.

It is therefore relevant to investigate the further development of vertebrate SIATs for sialyllactose production and possibly other niche markets. Most commercial SIATs are mammalian in origin, thus the enzymes have adapted to function in an environment of constant temperature, $\mathrm{pH}$ and salinity. As a result, the enzymes are not necessarily optimal for in vitro reactions. On the other hand, non-mammal species function in much more variable environments and their enzymes will therefore be more variable in their kinetic parameters, substrate specificities and stabilities (Siddiqui \& Cavicchioli 2006). For example, fishes live in conditions of high variability of temperature $\left(0-40^{\circ} \mathrm{C}\right)$ and salinity (freshwater, brackish or seawater). 
86 There is little information on the kinetics and substrate specificities of vertebrate non-

87 mammalian sialyltransferases. In addition, an understanding of the specificity of non-mammalian

88

89

90

91

92

93

94

95

96

97 enzymes to substituted Sia donors would be invaluable in designing new oligosaccharide-based drug analogues. This will become increasingly important as sialosides are targeted in clinical applications (Bauer \& Osborn 2015).

SIATs are subdivided into the ST6Gal, ST6GalNAc, ST3Gal and ST8Sia families defined by the sugar acceptor (Gal, GalNAc or Sia) or the specific linkage $(\alpha 2,3 ; \alpha 2,6$ or $\alpha 2,8)$ (Harduin-Lepers et al. 2005). The SIATs are classical type II membrane proteins residing in the Golgi body. The C-terminal catalytic domain constitutes the bulk of the protein, faces the Golgi lumen and is tethered to the Golgi membrane by a short N-terminal transmembrane sequence. A mostly unstructured stem connects the transmembrane anchor and the catalytic domains. The sequence homology across all the sialyltransferase proteins is strong only in very specific regions (L, S, III and VS motifs) within the catalytic domain, although within any specific family the homology extends to additional motifs (Harduin-Lepers 2010). The L-motif, associated with donor (CMPNeu5Ac) binding, is not surprisingly well conserved across SIATs as all use the same donor (Datta \& Paulson 1995). Although it was once thought that bacterial SIATs were unrelated to animal SIATS, it is now evident that there is low level amino acid homology at least in the Land S-motifs, and definite structural homology (Meng et al. 2013; Rao et al. 2009). The crystal structures of pig ST3Gal I (Rao et al. 2009), human ST6Gal I (Kuhn et al. 2013), rat ST6Gal I (Meng et al. 2013), human ST8Sia III (Volkers et al. 2015), human ST6GalNAc II (Moremen et al. 2018) and several bacterial sialyltransferases have been recently determined (see review (Audry et al. 2011)). In evolutionary terms ST6Gal enzymes exist in all levels of the animal kingdom back to the echinoderms and hemichordates (Petit et al. 2018). In higher animals both 
109 ST6GAL1 and ST6GAL2 paralogs exist, however, ST6Gal II has a much more restricted tissue

110 distribution than ST6Gal I which is ubiquitously expressed. The earliest ST3Gal enzymes are

111 found in echinoderms (Lehmann et al. 2008) and sponges (Petit et al. 2015). There are at least

112 eight ST3Gal paralogs (Teppa et al. 2016) with different kinetics and substrate specificities.

113 The rat, human, and mouse enzymes are probably the best kinetically characterised of the

114 sialyltransferases. However they are difficult enzymes to assay directly and there are many

115 natural acceptor substrates (oligosaccharides and glycoconjugates) and several potential Sia

116 donors that could be assayed. Sia is a generic term for substituted derivatives of the nine-carbon

117 sugar neuraminic acid. The major Sia donors of biological significance are Neu5Ac ( $N$ -

118 acetylneuraminic acid), Neu5Gc ( $N$-glycolylneuraminic acid) and Kdn (2-keto-3-deoxy-D-

119 glycero-D-galacto-nononic acid) but probably the most clinically relevant is Neu5Gc. Neu5Gc is

120 not synthesised in humans because the enzyme that converts Neu5Ac to Neu5Gc is absent.

121 However, other animals make Neu5Gc therefore ingested meat, xenograph transplants and

122 therapeutic proteins produced by animal cells in culture expose humans to this sialic acid. The

123 human immune system has been shown to recognise as foreign glycoconjugates containing

124 Neu5Gc.

125 We have cloned SIAT genes from a variety of vertebrate species and expressed these as secreted

126 proteins (ST6Gal I and ST3Gal IV) in CHO cells. The tagged and purified proteins were assayed

127 for activity in a phosphate-linked assay, for ability to bind appropriate lectins and for production

128 of sialyllactoses.

129

130 MATERIALS AND METHODS

131 Materials 
132 All materials were purchased from Sigma-Aldrich unless otherwise stated. Vectors

$133 \mathrm{pCR} 8 / \mathrm{GW} / \mathrm{TOPO}$ and pSecTag/FRT/V5-His-TOPO, and the Gateway (GW) Vector Conversion

134 System were obtained from Invitrogen. LR Clonase II and the Flp-In-CHO cell line were also

135 from Invitrogen. FITC-labelled lectins SNA-I and MAA were from EY Laboratories and

136 biotinylated SNA-I, RCA-I and MAA lectins were from Vector Laboratories. Oligonucleotide

137 primer synthesis and DNA sequencing was carried out by Eurofins. Proof-reading Phusion High-

138 Fidelity DNA Polymerase (New England Biolabs) was used for PCR amplification of cDNA

139 during construction and standard Taq polymerases (Promega) for screening. PNGase F was from

140 New England Biolabs.

141 Construct design

142 Protein sequences (Table 1) were aligned in MEGA-X (Figure S1). Only the soluble, 143 cytoplasmic catalytic domains of the sialyltransferases were cloned because we wanted these

144 proteins to be secreted into the media. In order to make all the cloned proteins as comparable as 145 possible we defined the catalytic domains of ST6Gal I and ST6Gal II as starting 121 amino acids

146 N-terminal to a Cys that is totally conserved across all vertebrate sialyltransferases, and which is 147 part of a disulfide bridge that links the L- and S-motifs (Cys-184 in human ST6Gal I

148 NP_003023.1) (Figure 1). In addition there will be linking amino acids as a consequence of the 149 recombination sequences that are part of the Gateway cloning system (Table S1). Most proteins 150 have identical sequences through their N-terminal fusions to the SIAT catalytic domain (ending 151 SEFAL). Slight variations in ST6Gal I (zebrafish) fusion sequences ending at KLAL, ST6Gal I 152 (stickleback) ending at AGS and ST3Gal IV (zebrafish) with an additional QKKW sequence are 153 a consequence of an evolving cloning approach (Table S1) and are explained further below. For 154 comparative purposes we have also constructed full-length human and zebrafish ST6Gal I 
155 expression vectors. These constructs, based on pcDNA5/FRT/V5-His-TOPO (Invitrogen), do not

156 support active secretion from CHO cells, however, the catalytic domain of ST6Gal I is detected

157 in cell culture supernatants (Donadio et al. 2003; Monaco et al. 1996).

158 Human ST6GAL1 cDNA was sourced from the HepG2 cell line while human ST6GAL2 was

159 sourced from the RPMI cell line. Other STGAL cDNAs were isolated from species include

160 mammals - rat (Rattus norvegicus); birds - chicken (Gallus gallus); fish - fugu (Takifugu

161 rubripes), stickleback (Gasterosteus aculaeatus) and zebrafish (Danio rerio). Total RNA (2 $\mu \mathrm{g})$

162 was isolated from appropriate tissues or cells using the RNeasy Kits (Qiagen) following the

163 manufacturer's recommended protocol (Table S2). Contaminating genomic DNA was removed

164 using an on-column DNase I treatment using an RNase-free DNase Kit (Qiagen). RNA was

165 reverse-transcribed using Superscript VILO (Invitrogen) as detailed in the manufacturer's

166 protocol.

\section{Cloning}

168 Primers were designed to each SIAT cDNA ensuring that, after recombination, the gene would

169 be fused in-frame to the $\mathrm{N}$-terminal Igא secretion signal sequence as well as to the C-terminal

170 V5-His epitope and tag. SIAT fragments were amplified by PCR from cDNA, gel purified and

171 ligated into a Gateway (GW) entry vector (pCR8/GW/TOPO). The Gateway Vector Conversion

172 System (Invitrogen) was used to convert pSecTag/FRT/V5-His-TOPO to a corresponding

$173 \mathrm{pSecTag} / \mathrm{FRT} / \mathrm{GW}$ destination vector. In brief, a GW cassette containing attR recombination

174 sites flanking a $c c d$ B gene and a chloramphenicol-resistance gene, was amplified by PCR using

175 specific primers and ligated into pSecTag/FRT/V5-His-TOPO vector. Ligations were

176 transformed into $c c d \mathrm{~B}$ survival competent cells, allowing propagation of the GW destination

177 vector containing the $c c d \mathrm{~B}$ gene. SIAT cDNA fragments were then transferred to this destination 
178

179

180

181

182

183

184

185

186

187

188

189

190

191

192

193

194

195

196

197

198

199

200

vector (pSecTag/FRT/GW) by LR recombination. LR Clonase II was used to catalyse the in vitro recombination between the entry GW clone (containing SIAT flanked by attL sites) and the GW destination vector (containing attR sites). After recombination these sites become attB1 and attB2 sites. Clones were sequenced to confirm in-frame fusion with the N-terminal leader and Cterminal tags. As noted above, there were some variations to this routine procedure leading to some linking sequence differences. Zebrafish st6gal1 was cloned directly into the pSecTag/FRT/V5-His-TOPO, and stickleback ST6GAL1 was recombined into our pSecTag/GW destination vector from a PCR-generated product containing added recombination sites in a related procedure (Fu et al. 2008). Either this led to the absence of a Gateway sequence in the zebrafish ST6Gal I fusion protein or a shorter Gateway sequence in the stickleback ST6Gal I protein. Zebrafish ST3Gal IV, for unknown reasons, included an additional short sequence which fortunately maintained reading frame.

\section{Transfection and culture of FIp-In-CHO cells and generation of stable cell lines}

Flp-In-CHO cells (Invitrogen) were grown in Ham F12, 10 \% FBS, L-glutamine (2 mM) containing $100 \mu \mathrm{g} / \mathrm{mL}$ zeocin. For stable transfections we used a 9:1 (w/w) ratio of pOG44 (1.8 $\mu \mathrm{g})$ (Invitrogen) to vector $(0.2 \mu \mathrm{g})$ and a 1:3 ratio of total DNA $(2 \mu \mathrm{g})$ to Fugene $6(6 \mu \mathrm{L})$ (Promega). The plasmid pOG44 expresses the Flp recombinase that recombines the pSecTag derivative plasmid FRT sites with the corresponding sites in the Flp-In-CHO cell chromosome. Transfections were carried out in 6-well plates (Nunc) containing respectively $3 \times 10^{5}$ cells.

Media was removed from adherent, low passage number Flp-In-CHO cells at approximate $60 \%$ confluence and replaced with fresh Ham F12, $10 \%$ FBS, 2 mM L-glutamine in the absence of zeocin. After $24 \mathrm{~h}$ incubation at $37{ }^{\circ} \mathrm{C}$ media was replaced with fresh Ham F12, $10 \%$ FBS, Lglutamine $(2 \mathrm{mM})$ containing $600 \mu \mathrm{g} / \mathrm{mL}$ hygromycin (optimised previously). Media was 
201 changed every 2 days until foci appeared at approximately day 10 (approximately $99 \%$ of non-

202 transfected Flp-In-CHO cells were dead at this stage). At 3 weeks, cells were rinsed with PBS,

203 treated with TrypLE (Gibco) under standard procedures, resuspended with 2 mL Ham F12, $10 \%$

204 FBS, 2 mM L-glutamine, $600 \mu \mathrm{g} / \mathrm{mL}$ hygromycin and transferred to a T25 flask containing 10

$205 \mathrm{~mL}$ of media. These cells were subsequently split every 3-4 days to T75 flasks and stocks were

206 frozen (liq. $\mathrm{N}_{2}$ ) at passage 5. Once stable lines were established, Flp-In-CHO cells were weaned

207 into ProCHO-AT (Lonza) containing $150 \mu \mathrm{g} / \mathrm{mL}$ hygromycin for several passages over 4-6

208 weeks. Cells grew strongly in ProCHO-AT but with both adherent and suspension phenotypes.

209 For upscaling, cells were split and grown in T175 triple flasks $\left(500 \mathrm{~cm}^{2}\right)$ in ProCHO-AT to

210 provide $1 \mathrm{~L}$ of media for experiments $(5 \times 200 \mathrm{~mL})$.

\section{Lectin Microscopy}

212 Cells were seeded ( $3 \mathrm{~mL}, 1.2 \times 10^{6}$ cells) in 6-well plates containing sterile coverslips and

213 incubated under standard $\mathrm{CHO}$ culture conditions to approximately $60 \%$ confluence. PBS-

214 washed cells were fixed with $4 \%$ paraformaldehyde. Cells were washed 3 times with a modified

215 TBS buffer, mTBS (20 mM Tris-HCl, pH 7.2, $100 \mathrm{mM} \mathrm{NaCl}, 1 \mathrm{mM} \mathrm{MgCl} 2,1 \mathrm{mM} \mathrm{CaCl}_{2}$ ),

216 blocked with $1 \%$ BSA (Sigma, $\geq 99 \%$, globulin free) for $30 \mathrm{~min}$ then washed as before with

217 mTBS. FITC-labelled lectins (EY Laboratories) SNA-I and MAA (containing both MAA-1 and

218 MAA-2) were prepared in mTBS $(40 \mu \mathrm{g} / \mathrm{mL})$, added to coverslips $(0.5 \mathrm{~mL}, 20 \mu \mathrm{g})$ and incubated

219 for $2 \mathrm{~h}$ at $4{ }^{\circ} \mathrm{C}$ in the dark in the absence or presence of $1 \mathrm{~mL} 100 \mathrm{mM}$ lactose. Plates were

220 washed 3 times with mTBS and then with PBS. The coverslip with the attached cells was

221 mounted on a glass slide with one drop of ProLong Gold antifade mountant containing DAPI

222 (Thermo Fisher Scientific) and allowed to dry overnight in the dark. Images were acquired using 
223 an upright microscope (Olympus BX53) with violet (autofluorescence/DAPI) and green (FITC)

224 filters.

\section{Sialyltransferase assay}

226 The Sialyltransferase Activity Kit (R\&D Systems) was used essentially as described by the

227 manufacturer and as described elsewhere (Meng et al. 2013; Wu et al. 2011). Our standard assay 228 conditions, used for comparative purposes across a range of sialyltransferase preparations, were 229 MES, pH 6.5 (100 mM), $\mathrm{MnCl}_{2}(10 \mathrm{mM}), \mathrm{CD} 73$ (50 ng), CMP-Neu5Ac (donor), acceptor and 2 $230 \mu \mathrm{L}$ sialyltransferase in a total volume of $25 \mu \mathrm{L}$. All assays were carried out in triplicate.

231 Acceptors were lactose $(2.6 \mathrm{mM})$ or $N$-acetyllactosamine (LacNAc, $2.6 \mathrm{mM})$. Routinely we used

$2320.2 \mathrm{mM}$ CMP-Neu5Ac donor for comparative assays and $1 \mathrm{mM}$ CMP-Neu5Ac (with $8 \mathrm{mM}$

233 lactose) when determining accurate specific activities ( $4 \mathrm{x} \mathrm{K}_{\mathrm{m}}$ concentrations). The

234 sialyltransferase was $100 \mathrm{ng}$ commercial of ST6Gal I (Sino Biologicals (mouse)) or varying

235 amounts of crude and purified sialyltransferase preparations. Negative control was purified

236 prostate specific antigen (PSA) produced in parallel, originally from the pSecTag/FRT/V5-His-

237 TOPO Vector Kit. Reactions were incubated for $20 \mathrm{~min}$ or $30 \mathrm{~min}$ at $37^{\circ} \mathrm{C}$. For specific activity

238 determination a range of enzyme amounts were assayed while keeping both donor and acceptor

239 levels at non-limiting levels.

\section{Purification}

241 Bovine serum albumin present in FBS was a contaminant in early purification protocols

242 therefore Flp-In-CHO cells were weaned onto ProCHO-AT media. Cell-free media (50 mL) in

243 1x binding buffer $(50 \mathrm{mM}$ Tris- $\mathrm{HCl}, \mathrm{pH} 7.5,500 \mathrm{mM} \mathrm{NaCl})$ and $1 \mathrm{~mL} \mathrm{Ni}$ Sepharose Excel resin

244 (GE Healthcare) were incubated overnight on a roller at $4{ }^{\circ} \mathrm{C}$. The resin was collected after

245 centrifugation $\left(4000 \mathrm{~g}, 15 \mathrm{~min}, 4^{\circ} \mathrm{C}\right)$ and packed into columns. Columns were washed with 100 
246 column volumes (CV) binding buffer containing $20 \mathrm{mM}$ imidazole (optimised for protein yield

247 and sialyltransferase activity across a 0-30mM imidazole range). Binding proteins were eluted

248 with $2 \mathrm{CV} 50 \mathrm{mM}$ Tris-HCl, $\mathrm{pH}$ 7.5, $500 \mathrm{mM} \mathrm{NaCl}$ containing $500 \mathrm{mM}$ imidazole. Buffer

249 exchange with TBS (20 mM Tris-HCl, $\mathrm{pH} 7.2,100 \mathrm{mM} \mathrm{NaCl})$ was carried out using $3 \mathrm{kDa}$ spin

250 filters (Amicon) by centrifugation at $4000 \mathrm{~g}$ for $30 \mathrm{~min}$ at $4{ }^{\circ} \mathrm{C}$ ( 5 times). The retentate

251 (approximately $500 \mu \mathrm{L}$ ) was concentrated using a $30 \mathrm{kDa}$ spin filter to approximately $50 \mu \mathrm{L}$.

252 Protein concentrations were measured spectrophotometrically by NanoDrop and calibrated

253 against BSA protein standards using the BCA protein assay (Pierce). Proteins were analysed in

254 reducing conditions on $12 \%$ NuPAGE Novex Bis-Tris gels using the NuPAGE MOPS SDS

255 Buffer Kit (Thermo Fisher Scientific). The predicted molecular weights of all secreted

256 sialyltransferase fusion proteins are given in Table S3. Gels were Coomassie or silver stained. A

257 wet transfer protocol to PVDF membrane (1 h at $24 \mathrm{~V}$ ) was used (Invitrogen). Blocking was with

$2584 \%$ dried skimmed milk (Marvel) in TBS-T (TBS $+0.05 \%$ Tween-20) overnight at $4{ }^{\circ} \mathrm{C}$.

259 Detection of His-tagged proteins used either a two-step protocol with mouse anti-His antibody

260 (Novagen, 1:1000 dilution) and a HRP-conjugated goat anti-mouse IgG (Pierce/Thermo

261 Scientific, 1:1000 dilution), or a one-step protocol using mouse anti-His-HRP (Sigma, 1:1000

262 dilution). BenchMark His-tagged Protein Standard and PageRuler Prestained Protein Ladder (10

263 to $180 \mathrm{kDa}$ ) were loaded as molecular weight markers (Thermo Fisher Scientific). Detection was

264 either with DAB (chromogenic) or with ECL (Advansta). Where applied Ni Sepharose-purified

265 protein was digested with PNGase F (New England Biolabs) for $1 \mathrm{~h}$ at $37^{\circ} \mathrm{C}$ as recommended by

266 the manufacturer.

267 HILIC-HPLC 
268 Glycans were labelled with 2-amino benzamide (2-AB)(Bigge et al. 1995) and separated by

269 hydrophilic interaction liquid chromatography (HILIC) using a GlycoSep N-Plus HPLC column

270 (Prozyme) on a Waters Alliance 2695 HPLC system with Waters 2475 fluorescence detection.

271 Flow rate was $0.67 \mathrm{~mL} / \mathrm{min}$ with $20 \% 50 \mathrm{mM}$ ammonium formate, $\mathrm{pH} 4.4$ (solvent A) and $80 \%$

272 acetonitrile (Romyl). Samples were injected in $80 \%$ acetonitrile with a linear gradient of 20-58

$273 \%$ solvent A over 48 min. Sialyltransferase reactions ( $50 \mu \mathrm{L}$ scale) were carried out much as

274 described above using excess of donor (CMP-Neu5Ac) and acceptor (LacNAc, Lac or whey

275 permeate) at approximately $4 \mathrm{x} \mathrm{K}_{\mathrm{m}}$ concentrations. Whey permeate (provided by Glanbia plc)

276 was $79.6 \%$ lactose by our estimate. Approximately $900 \mathrm{ng}$ of all sialyltransferases (controls and

277 experimental samples) were assayed. Duration $(4 \mathrm{~h}$ or $16 \mathrm{~h})$ and temperature $\left(37^{\circ} \mathrm{C}\right.$ or $\left.20^{\circ} \mathrm{C}\right)$ of

278 incubations were varied though $16 \mathrm{~h}$ incubation became routine. After reaction $450 \mu \mathrm{L}$ ice-cold

279 HPLC-grade water was added to the reaction, followed by centrifugation (10 min, 20,000 g, 4

$\left.280{ }^{\circ} \mathrm{C}\right)$ through Amicon spin filters. Eluants were concentrated to dryness ready for 2-AB labelling.

281 Resulting glycans were fluorescently labelled with 2-AB by reductive amination (Bigge et al.

282 1995). In brief, an appropriate volume of 0.35 M 2-AB in DMSO containing $30 \%$ acetic acid

283 was dissolved in sodium cyanoborohydride $(1 \mathrm{M})$ at $65^{\circ} \mathrm{C}$. A volume of $60 \mu \mathrm{L}$ of the preparation

284 was used to label dried glycans. Samples were incubated for $2.5 \mathrm{~h}$ at $65^{\circ} \mathrm{C}$. A GlycoClean $\mathrm{S}$

285 cartridge (Prozyme) was used to separate labelled glycans from unreacted 2-AB following the

286 manufacturer's instructions. Elution was carried out with $3 \times 0.5 \mathrm{~mL}$ water and 2-AB labelled

287 glycans were evaporated to dryness using a concentrator. In this HILIC system each sugar has a

288 retention time, stated in glucose units $(\mathrm{GU})$ that is unique for that molecule. Calibration of

289 retention with dextran $(20 \mu \mathrm{g}$, dextran ladder from Leuconostoc mesenteroides, Fluka) was

290 carried out three times over the course of a run to insure accurate GU values. Fifth polynomial 
291 equation resolution (GraphPad Prism) was used to convert retention times into GU (Marino et al.

292 2011). Calibration of quantities was determined for a 10-fold dilution (1 $\mu \mathrm{g}$ to $0.1 \mathrm{ng}$ ) of lactose,

$2933^{\prime}$ sialyllactose and 6' sialyllactose using peak height and/or integrated area. Standards were

294 LacNAc (CarboSynth), Lac (Sigma), 3' sialyllactose (Sigma), 6' sialyllactose (Glycom) and

295 Neu5Ac (Sigma).

296 Enzyme linked lectin assay (ELLA)

297 ELLA was based on previous protocols (Couzens et al. 2014; McCoy et al. 1983; Thompson et

al. 2011). Lectin binding is generally quite weak and requires multivalent binding sites acting

299

300

301

302

303

304

305

306

307

308

309

310

311

312

313 together to give strong and specific binding. For this assay, we therefore used asialofetuin (ASF) as the acceptor in preference to the disaccharides Lac or LacNAc. Fetuin is a simple glycoprotein (Figure S2) with a defined structure offering six potential terminal LacNAc sites and is readily available in its asialylated form. Asialofetuin (ASF) $(50 \mu \mathrm{L}$ per well of a $0.5 \mu \mathrm{g} / \mathrm{mL}$ solution in PBS) was added to Nunc ELISA plates (Thermofisher MaxiSorp Polystyrene) and incubated at 4 ${ }^{\circ} \mathrm{C}$ overnight. Wells were blocked for $2 \mathrm{~h}$ at room temperature with $150 \mu \mathrm{L} 0.5 \%(\mathrm{w} / \mathrm{v})$ polyvinyl alcohol (PVA). Wells were washed (4 times) with $100 \mu \mathrm{L}$ mTBS-T (mTBS containing $0.05 \%$ Tween 20$)$. Sialyltransferase reactions $(50 \mu \mathrm{L})$ were prepared containing 50mM MES pH 6.5, 10mM $\mathrm{MnCl}_{2}, 0.2 \mathrm{mM} \mathrm{CMP-Neu5Ac,} 2.5$ units Antarctic phosphatase (New England Biolabs) and the sialyltransferase preparation to be assayed. We used 100, 200 and $400 \mathrm{ng}$ of commercial mouse ST6Gal I as positive control, $400 \mathrm{ng}$ of our test sialyltransferase or a negative control with no sialyltransferase. Reactions was added to wells and incubated at $37{ }^{\circ} \mathrm{C}$ for $16 \mathrm{~h}$. Wells were washed (4 times) with $100 \mu \mathrm{L}$ mTBS-T and biotinylated lectins (Vector Laboratories) were added at 2, 5 or $10 \mu \mathrm{g} / \mathrm{mL}$ of SNA-I, RCA-I or MAA respectively. After a further $1 \mathrm{~h}$ incubation at room temperature wells were again washed (4 times) with $100 \mu \mathrm{L}$ 
314 mTBS-T. HRP-Streptavidin (R\&D Systems) was added at 1/200 dilution and incubated $1 \mathrm{~h}$ at

315 room temperature. Wells were washed (4 times) with $100 \mu \mathrm{L}$ mTBS-T. TMB substrate $(50 \mu \mathrm{L})$

316 was added followed by incubation at room temperature for $15 \mathrm{~min}$ with the reaction stopped by

317 adding $50 \mu \mathrm{L} 2 \mathrm{M}$ sulfuric acid. Absorbance was measured at $450 \mathrm{~nm}$ (SpectraMax® M5,

318 Molecular Devices).

319 Statistics

320 Student $t$-tests were used to compare SIAT activities between recombinant PSA produced by the

321 control Flp-In-CHO cell line and all other recombinant sialyltransferases purified from stable cell

322 lines. Mann-Whitney tests were carried out to compare the lectin binding of ASF with all

323 recombinant sialyltransferases. Tests were performed with PASW Statistics 22 (SPSS, USA).

\section{RESULTS}

325 Constructs

326 All known sialyltransferases (CAZy-family 29) have an approximate 250 amino acid catalytic

327 domain that is naturally orientated to the lumen of the Golgi. The full length protein has a

328 transmembrane domain that attaches the catalytic domain to the Golgi membrane through a stem

329 structure that is not essential for catalytic activity. Our focus in this study was on expressing the

330 catalytic domains of the sialyltransferases, however, for comparative purposes we also

331 constructed two full-length ST6Gal I expressing vectors (see Materials and Methods). All

332 catalytic domain-expressing constructs were based on pSecTag/FRT/V5-His TOPO with inserts

333 amplified by PCR from cDNA. In order to make all the cloned proteins as comparable as

334 possible we aligned all the protein sequences and maintained a consistent length peptide linker

335 (part of the stem) between the Igא secretion signal sequence and the known functional limits of

336 the catalytic domain. ST6Gal I and ST6Gal II therefore started 121 amino acids N-terminal to a 
337 Cys that is totally conserved across all animal sialyltransferases (Cys-184 in human ST6Gal I).

338 The first amino acid in the human ST6Gal I was Val-63. For ST3Gal IV we chose to define the

339 catalytic domain as starting approximately 70 amino acids $\mathrm{N}$-terminal to this same invariant Cys.

340 All ST6Gal I, ST6Gal II and ST3Gal IV expressing constructs are summarized in Table 1 and

341 Figure 1, with additional details in Tables S1-S3. Some minor differences that predated our final

342 routine cloning approach are explained in the Materials and Methods section.

343 Sequences

344 We cloned and developed stable cell lines of three separate human ST6Gal I sequences. One was

345 the wild-type sequence (NP_003023.1). Clone hST6 B1 had an interesting G327S mutation in a

346 highly conserved region in the S-motif: this Gly is highly conserved across ST6Gal I and ST6Gal

347 II proteins in all species (Harduin-Lepers 2010; Harduin-Lepers et al. 2005). Clone hST6 B1

348 subsequently refers to the codon-optimised and synthesised version of this clone. hST6 A5 had

349 two mutations (I295T and K395R) in less highly conserved regions. The zebrafish clone zST6

350 had both an H111I and a K187R mutation in comparison to the database sequence

351 (NP_001003853.1). Rat had a R105G mutation. All other ST6Gal I protein sequences matched

352 the database sequences (chicken, fugu and stickleback). Human ST6Gal II (sourced from the

353 RPMI cell line) had an A269T missense mutation. Human ST3Gal IV clones (hST3 A1 and

354 hST3 A2) were different by a six amino acid insertion present in hST3 A1 but were otherwise

355 identical to the database protein sequences. Some of these variant sequences may have been

356 inadvertently introduced in the amplification process (though proof-reading Taq polymerases

357 were used throughout for cloning) or be common variants in laboratory species, but whatever

358 their origins they were useful for the purposes of this study which was to generate a library of

359 enzymes with potentially different physiochemical properties. 
360 Clones were transfected into Flp-In-CHO cells and selected for with hygromycin. Transfected

361 cDNA should recombine at the corresponding FRT sites engineered into the CHO chromosome

362 of these Flp-In cells, therefore no further selection was carried out to identify highly expressing

363 clones and transfected cells were pooled. The transfected cells were investigated initially for

364 lectin binding. CHO cells do not express ST6Gal I therefore there are minimal NeuAc( $\alpha 2-6) \mathrm{Gal}$

365 terminal structures on surface glycans (Jenkins et al. 1996; Lee et al. 1989; Monaco et al. 1996).

366 The Sambucus nigra SNA-I lectin has specificity for NeuAc( $\alpha 2-6)$ Gal/GalNAc therefore this

367 lectin can be used to identify terminal $\mathrm{NeuAc}(\alpha 2-6)$ structures present in $\mathrm{CHO}$ cells as a result of

368 functional expression of the transfected cDNA (Smith et al. 2010). The Maackia amurensis

369 MAA lectin (undefined mixture of MAA-1 and MAA-2) on the other hand has specificity for

370 NeuAc( $\alpha 2-3)$ Gal/GalNAc (Geisler \& Jarvis 2011) therefore this lectin can be used to identify

371 terminal NeuAc( $\alpha 2-3)$ which is naturally produced in CHO cells by the activity of ST3Gal

372 enzymes. We have used these two lectins to show that $\operatorname{NeuAc}(\alpha 2-6)$ structures are much more

373 prominent in transfected CHO cells (ST6GAL1-transfected) and that there is little changes in the

$374 \operatorname{NeuAc}(\alpha 2-3)$ structures (Figure 2).

375 SIAT assays by phosphate linked assay

376 Sialyltransferase activity was determined indirectly through a phosphate linked assay. LacNAc is

377 considered the best acceptor for human ST6Gal I in this type of assay. Suitable potential

378 acceptors were LacNAc, asialofetuin (ASF) or Lac. As production of sialyllactose was the focus

379 of our study, Lac or LacNAc (as disaccharides) were preferred over ASF (a glycoprotein).

380 Samples assayed were predominantly His-purified proteins isolated from the media of stably

381 transfected cells, though initially cell lysates were used instead of secreted protein. We

382 determined significant sialyltransferase activity for purified ST6Gal I proteins of all species 
383 which were comparable to the commercial control (mouse ST6Gal I, mST6 in Figure 3). Both

384 mutant human ST6Gal I proteins (hST6 B1 and hST6 A5) were active. Of the ST3Gal IV

385 preparations, only zebrafish ST3Gal IV (zST3) showed significant activity. The two human

386 ST3Gal IV clones (hST3 A1, hST3 A2) and the human ST6Gal II clone showed marginal

387 activity in this assay. The results may be influenced by the choice of acceptor: it is possible that

388 these constructs, showing marginal activity with LacNAc, would show stronger activity with

389 ASF as acceptor. For several enzymes we also measured specific activity across a range of

390 protein amounts. In a direct comparison of activity in cellular lysates human ST6Gal I clones

391 hST6 A5, hST6 B1 and zebrafish clone zST6 gave activities of $0.6 \mathrm{pmol} / \mathrm{min} / \mu \mathrm{g}, 7.1$

$392 \mathrm{pmol} / \mathrm{min} / \mu \mathrm{g}$ and $6.2 \mathrm{pmol} / \mathrm{min} / \mu \mathrm{g}$ respectively. In these samples activity may be influenced by

393 the efficiency of secretion with a poor secretor giving a proportionally higher level of protein in 394 the lysates.

395 Protein Purification

396 Proteins were purified as described in Materials and Methods. Because of purification issues

397 around bovine serum albumin present in FBS, cells were weaned onto a serum-free media

398 (ProCHO-AT). A consequence of the switch to ProCHO-AT was that the CHO cells were not

399 fully adherent to culture plates. This was irrespective of whether the Flp-In-CHO cells were

400 transfected or not. Although we did not determine whether the two phenotypes (adherent and

401 non-adherent) differed in their recombinant sialyltransferase expression characteristics, we did

402 show that the phenotype was not fixed - non-adherent cells returned to a mix of adherent and

403 non-adherent cells when reseeded.

404 Typical protein yields from $200 \mathrm{~mL}$ media ranged from 1-33 $\mu \mathrm{g}$ across clones. This

405 corresponded to a typical recovery of 2-5\% though estimates of protein concentration in crude 
406 media were inconsistent. For comparative purposes sialyltransferase assays were carried out with

407 a fixed volume of recombinant enzyme and corrected for protein concentration. Specific

408 activities for select preparations of cell lysates, media or IMAC-purified proteins were

409 determined across a range of protein amounts. Specific activities of $1697 \mathrm{pmol} / \mathrm{min} / \mu \mathrm{g}$ (for

410 commercial mouse ST6Gal I) and $3147 \mathrm{pmol} / \mathrm{min} / \mu \mathrm{g}$ (for human ST6Gal I, hST6 A5, His-

411 purified from a cellular lysate) corresponded to activity measurements for both of approximately

$412450 \mathrm{pmol} / \mathrm{min} / \mu \mathrm{g}$ in the comparative assay (fixed protein) of Figure 3. Purified proteins were

413 silver-stained on SDS/polyacrylamide gels and were analysed on western blots using anti-His

414 antibodies (Figure 4A). Typically bands on gels were weak and varied in size depending on the

415 species. Glycosylation (heterogeneous) also led to diffuse bands for several proteins and the

416 suggestion of different prominent glycoforms. PNGase-F digestion of purified human ST6Gal I

417 showed a shift downward in molecular mass from $55 \mathrm{kDa}$ (for a prominent glycoform) to $41 \mathrm{kDa}$

418 (Figure 4B). There were two prominent glycoforms in this preparation of $55 \mathrm{kDa}$ and $43 \mathrm{kDa}$,

419 with two weaker glycoforms of $60 \mathrm{kDa}$ and $50 \mathrm{kDa}$.

$420 \underline{\text { HILIC }}$

421 Indirect evidence from the phosphate linked assay suggested the presence of sialyltransferase

422 activity. Direct evidence was obtained by incubating the enzyme with lactose and detecting the

423 expected products (3 sialyllactoses for ST3Gal IV and 6' sialyllactose for ST6Gal I). The

424 products were labelled with (2-AB) and separated by HILIC. Each sugar has a retention time,

425 which when stated in glucose units (GU) calibrated against a dextran ladder, is unique for that

426 molecule. Sialyllactose standards generated distinctive GU values of 2.88 and 3.35 for $3^{\prime}$ and $6^{\prime}$

427 sialyllactose respectively. 
428 Reactions were carried out routinely with $8 \mathrm{mM}$ lactose (or whey permeate equivalent) and 2

$429 \mathrm{mM}$ CMP-Neu5Ac which approximate to $4 \mathrm{x} \mathrm{K}_{\mathrm{m}}$ concentrations (for human ST6Gal I), ensuring

430 that both acceptor and donor were in excess. Yields were checked at $1 \mathrm{~h}, 4 \mathrm{~h}$ and $16 \mathrm{~h}$ at $37{ }^{\circ} \mathrm{C}$

431 but were subsequently set at $16 \mathrm{~h}$. Both $3^{\prime}$ and $6^{\prime}$ sialyllactoses peaks were evident at the

432 expected positions depending on the purified enzyme used, ST3Gal IV or ST6Gal I respectively

433 (Figure 5). The human ST6Gal I clone (hST6 B1) yielded $11.5 \mathrm{ng} 6$ ' sialyllactose considerably

434 higher than the 3.9 ng of 6' sialyllactose achieved with ST6Gal II. Chicken and stickleback

435 ST6Gal I were also shown to yield 6' sialyllactose. The efficiencies of these reactions were

436 generally low, never achieving more than a $6 \%$ conversion of lactose to sialyllactose, though

437 better yields could have been achieved with more optimisation.

438 Enzyme-linked lectin assays (ELLA)

439 At the cellular level (Figure 2) we demonstrated increased SNA-I labelling of CHO cells

440 expressing ST6Gal I. At the molecular level and in vitro this can be demonstrated by the de novo

441 sialylation of asialofetuin (ASF). ASF in a microplate assay should show limited binding to

442 SNA-I but after reaction with purified ST6Gal enzymes there should be increased labelling by

443 SNA-I. There should also be reduced binding to the exposed Gal groups in ASF now hidden as a

444 result of sialylation. Gal groups (Gala1-4GlcNAc and other Gal 1 1-related oligosaccharides)

445 typically bind the RCA I lectin. In two separate experiments we observed clear binding of SNA-I

446 by ELLA after reacting ASF with the different purified sialyltransferases (Figure 6A). Chicken

447 and rat ST6Gal I were as effective as the commercial (mouse) ST6Gal I for adding on SNA-I

448 recognised sugars (probably Neu5Ac( $\alpha 2-6)$ linked to Gal. The other ST6Gal I preparations were

449 less effective and the ST6Gal II preparation showed no activity in this assay. As expected the

450 ST3Gal IV preparations did not affect SNA-I binding as Neu5Ac( $\alpha 2-3)$ linked to Gal is not an 
451 epitope for SNA-I. Reduced binding of RCA I was also observed for the strongest ST6Gal I

452 preparations (Figure 6B). The most effective enzyme in this assay was chicken ST6Gal I with

453 approximately the same activity as the commercial mouse ST6Gal I.

\section{DISCUSSION:}

455 Sialyltransferases are very important enzymes that shape the terminal structures on glycoproteins

456 and glycolipids and can have profound effects on how cellular surfaces interact with each other

457 (Varki 2007). The enzymes are therefore key players in determining how a cell recognises a

458 foreign bacterium or virus. Sialyltransferases also modify free oligosaccharides: new-born

459 human milk contains high concentrations of oligosaccharides including the sialyllactoses that are

460 synthesised from lactose by ST6Gal and ST3Gal enzymes. These human milk oligosaccharides

461 offer protection against pathogens both directly in mopping up and excreting harmful bacteria

462 and indirectly in promoting the colonisation of health-promoting commensal bacteria (Bode

463 2012).

464 We have developed a panel of stable $\mathrm{CHO}$ cell lines that express a variety of vertebrate

465 sialyltransferases. Most commercial enzymes from non-bacterial sources are of mammalian

466 origin but we have chosen other species which have adapted to much more variable

467 environments (Table S2). We have included homeotherms such as humans with a blood

468 temperature of $36.5-37.5^{\circ} \mathrm{C}$, rats at $35.9-37.5^{\circ} \mathrm{C}$ and chickens at $40.6-41.7^{\circ} \mathrm{C}$. We have

469 included several fishes which are poikilotherms with body temperatures that will vary

470 considerably based on environmental conditions. Three-spined sticklebacks may live in water

471 temperatures of $0-29^{\circ} \mathrm{C}$ though optimal temperatures are considered $10-20{ }^{\circ} \mathrm{C}$ (Lefébure et al.

472 2011). Zebrafish are tropical fish, can survive temperatures of $6.2-41.7^{\circ} \mathrm{C}$ but their optimal

473 temperature is $18-28^{\circ} \mathrm{C}$ (Cortemeglia \& Beitinger 2005). Fugu are temperate fish and their 
474 optimal temperature is $15-24{ }^{\circ} \mathrm{C}$ (Nakajima et al. 2010). Furthermore, zebrafish are freshwater

475 fish where fugu and stickleback adapt to freshwater and saltwater conditions. Quite possibly

476 animals can survive at temperatures where their sialyltransferases are barely functional but it

477 would seem logical that the sialyltransferases work efficiently at the optimal growth temperature

478 of the animal. An isolated enzyme may however be more efficient at a different temperatures.

479 We have generated enzymes that are as comparable in structure to each other as possible by

480 defining, for our purposes, the start of the catalytic domain (Table 1). It has been reported that N-

481 terminal deletion between residues 90 and 100 in human ST6Gal I leads to a complete loss in

482 sialyltransferase activity (Donadio et al. 2003) though this may depend on the expression system

483 (Ribitsch et al. 2014). The full-length enzyme shows preference for some glycoproteins

484 acceptors over others but this preference is lost when the catalytic domain is untethered from the 485 membrane (Legaigneur et al. 2001). The untethered enzyme also shows increased activity as the 486 stem is reduced to approximately residue 60 when it increases no further (Legaigneur et al.

487 2001). We therefore chose to retain some structure in the stem region. However, it is possible 488 that in retaining more of the stem we have compromised protein stability as it is also known that 489 the stem is susceptible to protease attack (Kitazume-Kawaguchi et al. 1999). We also included 490 full-length human and zebrafish ST6Gal I sequences for comparative purposes. Both enzymes 491 were secreted in the tissue culture media and the cell lines displayed SNA-I binding following 492 lectin microscopy (Figure S4). Our approach to expressing recombinant ST3Gal IV was similar. 493 When ST3Gal I is deleted from the N-terminal from residue 57 to 77 enzymatic activity is lost 494 (Vallejo-Ruiz et al. 2001).

495 Sequencing of our constructs identified several mutations, both missense and silent. These were 496 most common in the human and zebrafish samples and rare in the other species, possibly as a 
497 consequence of long-term cultivation of the human (HepG2) and zebrafish sources in

498 laboratories worldwide. We used proof-reading Taq polymerases throughout and saw no

499 evidence that these mutations were introduced through amplification errors though this is still a

500 possibility. Although reports of overexpression of human ST6GAL1 in cancer are common

501 (Dall'Olio \& Chiricolo 2001; Kudo et al. 1998; Swindall et al. 2013), we are not aware of any

502 record of these specific mutations in ST6GAL1. The NCBI SNP Report (Sherry et al. 2001) for

503 human ST6GAL1 did however identify missense mutations directly adjacent to I295, I317, G327

504 and K395 which may suggest some accommodation for mutation in these particular regions.

505 Although the functional consequences of these SNPs are not known, we would expect functional

506 consequences for several of the mutations we identified as they were present in residues that are

507 highly conserved across species. The 18 nucleotide insertion in ST3GAL4 (clone A1) is a

508 recognised rare sequence so detection of this rare sequence variant in one of only two clones

509 sequenced is of interest (Grahn \& Larson 2001). It would be surprising if there is not a functional

510 consequence to this insertion as a potential $\mathrm{N}$-glycan attachment site (Asn) is removed.

511 Multiple sialyltransferase activity assay approaches have been reported but we chose to use an

512 indirect enzymatic approach for simplicity. Different acceptors (ASF, LacNAc or Lac) were

513 options but we focused on Lac (or LacNAc) because sialyllactose was our product of interest.

514 Specific activity and $\mathrm{K}_{\mathrm{m}}$ measurements have been reported for several sialyltransferases (native

515 and recombinant) using different approaches (Datta \& Paulson 1995; Legaigneur et al. 2001;

516 Ortiz-Soto \& Seibel 2016; Wu et al. 2011) and our assay protocol was planned based on these

517 values. Samples assayed were generally His-purified proteins from the media of stably

518 transfected cells. Although ST6Gal activity in $\mathrm{CHO}$ cells is undetectable due to non-expression

519 of the gene (Lee et al. 1989; Onitsuka et al. 2012) ST3Gal activity is present and will interfere in 
520 this assay system when unpurified proteins are used. There is no specific acceptor available that 521 can differentiate ST3Gal and ST6Gal activity in this assay. All ST6Gal I preparations of

522 sequence-confirmed constructs gave detectable sialyltransferase activity. The two human ST3Gal

523 IV clones and the human ST6Gal II clone showed marginal activity in this assay, though

524 zebrafish ST3Gal IV gave appreciable activity and human ST3Gal IV (clone A1) did produce 3'

525 sialyllactose as determined by HILIC-HPLC. There is speculation that ST6Gal II prefers free

526 oligosaccharides as acceptors over glycoproteins and glycolipids but lactose is not reported to be

527 a substrate (Krzewinski-Recchi et al. 2003; Takashima et al. 2002). Production of 3' sialyllactose

528 and 6' sialyllactose by incubation with the recombinant enzymes was confirmed by HILIC-

529 HPLC although yields were low.

530 We also used three lectins to confirm the ability of the recombinant proteins to add Neu5Ac onto

531 terminal Gal residues of ASF. Unfortunately biotin-labelled MAA binding was very low in this

532 assay format and we could not confirm definite specific binding to Neu5Ac( $\alpha 2-3)$ Gal structures

533 on ASF (though we had no ST3Gal commercial positive control). Chicken and rat ST6Gal I

534 enzymes yielded a glycoprotein that bound SNA-I much more strongly than ASF. As expected

535 the same preparations showed reduced binding to RCA-I. Other SIAT preparations were not as

536 effective but this could be a result of stability of the preparations or reduced affinity for ASF.

537 These stable cells may be used as a source of the purified sialyltransferase or they may be used to

538 sialylate in vivo recombinant proteins. In vivo sialylation will depend on the compatibility of the

539 growth conditions of the $\mathrm{CHO}$ cells expressing the recombinant protein of interest and the

540 specific ST6Gal or ST3Gal stably expressed. For example, a recombinant protein that is known

541 to be optimal in performance when produced at $30^{\circ} \mathrm{C}$ may best be paired up with the stable cell

542 line expressing a fish ST6Gal I whose activity is potentially favoured by lower temperatures. In 
543 vitro reaction with the purified protein will be less restrictive as conditions are not limited by the

544 growing CHO cell. Bacterial sialyltransferases are very efficient at modifying oligosaccharides

545 and, especially in so called 'one pot' reactions, are the preferred means of making human milk

546 oligosaccharides and many natural and unnatural sialosides. The promiscuity for modified donor

547 sialic acids and a variety of acceptors, together with their high level expression, makes the

548 bacterial enzymes ideal in this situation. However promiscuity is not an advantage when a

549 precise sialylated structure is required: here the vertebrate enzymes have a distinct advantage.

550 Yields of the purified proteins were low but there is still scope for optimisation. One major issue

551 that limited production was the low adherence phenotype of these $\mathrm{CHO}$ cells in serum-free

552 media. We have also not characterised the secretion profile of any construct in terms of the

553 optimal time to harvest the media. We have assumed that the FRT mechanism in the Flp-In-CHO

554 cells ensures high stable expression so we have not select maximal producers. Since the native

555 ST6GAL1 gene in CHO cells is not expressed it would be especially important to monitor the

556 stability of these cell lines over time in culture (Onitsuka et al. 2012). The recombinant enzymes

557 produced in this system have a direct effect on the host cells (as evidenced by lectin binding on

558 growing $\mathrm{CHO}$ cells in Figure 2) because the enzymes can potentially modify other cellular

559 glycoproteins and glycolipids in the $\mathrm{CHO}$ cells. This could have a significant effect on $\mathrm{CHO}$

560 growth characteristics, however, it is not the reason for the semi-adherent growth we observed.

561 We have not yet fully characterised any of these enzymes in relation to substrate preference, $\mathrm{K}_{\mathrm{m}}$

562 for substrates, temperature, salinity and pH optimums. Neu5Ac was the only sialic acid donor

563 used in our study as it is the major donor in humans, however, Neu5Gc and KDN are important

564 in other vertebrates. The expectation, based on their native environments, is that these proteins 
565

566

567

568

569

570

571

572

573

574

575

576

577

578

579

580

581

582

583

584

585

586

587

588

589

590

591

592

593

594

595

596

597

598

599

600

601

602

will show a range of biological and physiochemical characteristics which may be appropriate to

specific opportunities in the Biopharma sector.

\section{ACKNOWLEDGMENTS}

We would like to thank John O' Connor, Glanbia Ingredients Ireland for providing the whey

permeate.

\section{REFERENCES}

Audry M, Jeanneau C, Imberty A, Harduin-Lepers A, Delannoy P, and Breton C. 2011. Current trends in the structure-activity relationships of sialyltransferases. Glycobiology 21:716-726. 10.1093/glycob/cwq189

Bauer J, and Osborn HMI. 2015. Sialic acids in biological and therapeutic processes: opportunities and challenges. Future Medicinal Chemistry 7:2285-2299. 10.4155/fmc.15.135

Bigge JC, Patel TP, Bruce JA, Goulding PN, Charles SM, and Parekh RB. 1995. Nonselective And Efficient Fluorescent Labeling Of Glycans Using 2-Amino Benzamide And Anthranilic Acid. Analytical Biochemistry 230:229-238. 10.1006/abio.1995.1468

Bode L. 2012. Human milk oligosaccharides: Every baby needs a sugar mama. Glycobiology 22:11471162. 10.1093/glycob/cws074

Bragonzi A, Distefano G, Buckberry LD, Acerbis G, Foglieni C, Lamotte D, Campi G, Marc A, Soria MR, Jenkins N, and Monaco L. 2000. A new Chinese hamster ovary cell line expressing alpha 2,6sialyltransferase used as universal host for the production of human-like sialylated recombinant glycoproteins. Biochimica Et Biophysica Acta-General Subjects 1474:273-282. 10.1016/s03044165(00)00023-4

Choi YG, Park CJ, Kim HE, Seo YJ, Lee AR, Choi SR, Lee SS, and Lee JH. 2015. Comparison of backbone dynamics of the type III antifreeze protein and antifreeze-like domain of human sialic acid synthase. Journal of Biomolecular Nmr 61:137-150. 10.1007/s10858-014-9895-2

Cortemeglia C, and Beitinger TL. 2005. Temperature tolerances of wild-type and red transgenic zebra Danios. Transactions of the American Fisheries Society 134:1431-1437. 10.1577/T04-197.1

Couzens L, Gao J, Westgeest K, Sandbulte M, Lugovtsev V, Fouchier R, and Eichelberger M. 2014. An optimized enzyme-linked lectin assay to measure influenza $A$ virus neuraminidase inhibition antibody titers in human sera. Journal of Virological Methods 210:7-14. 10.1016/j.jviromet.2014.09.003

Dall'Olio F, and Chiricolo M. 2001. Sialyltransferases in cancer. Glycoconjugate Journal 18:841-850. 10.1023/a:1022288022969

Datta AK, and Paulson JC. 1995. The sialyltransferase "sialylmotif" participates in binding the donor substrate CMP-NeuAc. J Biol Chem 270:1497-1500. DOI 10.1074/jbc.270.4.1497

Donadio S, Dubois C, Fichant G, Roybon L, Guillemot JC, Breton C, and Ronin C. 2003. Recognition of cell surface acceptors by two human alpha-2,6-sialyltransferases produced in $\mathrm{CHO}$ cells. Biochimie 85:311-321. 10.1016/s0300-9084(03)00080-4 
603

604

605

606

607

608

609

610

611

612

613

614

615

616

617

618

619

620

621

622

623

624

625

626

627

628

629

630

631

632

633

634

635

636

637

638

639

640

641

642

643

644

645

646

647

648

Fierfort N, and Samain E. 2008. Genetic engineering of Escherichia coli for the economical production of sialylated oligosaccharides. Journal of Biotechnology 134:261-265.

10.1016/j.jbiotec.2008.02.010

Fu CL, Wehr DR, Edwards J, and Hauge B. 2008. Rapid one-step recombinational cloning. Nucleic Acids Research 36. 10.1093/nar/gkn167

Galuska SP, Oltmann-Norden I, Geyer H, Weinhold B, Kuchelmeister K, Hildebrandt H, Gerardy-Schahn R, Geyer R, and Muhlenhoff M. 2006. Polysialic acid profiles of mice expressing variant allelic combinations of the polysialyltransferases ST8Siall and ST8SialV. Journal of Biological Chemistry 281:31605-31615. 10.1074/jbc.M606516200

Geisler C, and Jarvis DL. 2011. Letter to the Glyco-Forum: Effective glycoanalysis with Maackia amurensis lectins requires a clear understanding of their binding specificities. Glycobiology 21:988-993. 10.1093/glycob/cwr080

Grahn A, and Larson G. 2001. Identification of nine alternatively spliced alpha 2,3-sialyltransferase, ST3Gal IV, transcripts and analysis of their expression by RT-PCR and laser-induced fluorescent capillary electrophoresis (LIF-CE) in twenty-one human tissues. Glycoconjugate Journal 18:759767. 10.1023/a:1021199300718

Harduin-Lepers A. 2010. Comprehensive analysis of sialyltransferases in vertebrate genomes. Glycobiology Insights 2:29-61. 10.4137/gbi.s3123

Harduin-Lepers A, Mollicone R, Delannoy P, and Oriol R. 2005. The animal sialyltransferases and sialyltransferase-related genes: a phylogenetic approach. Glycobiology 15:805-817. 10.1093/glycob/cwi063

Hennet T, Chui D, Paulson JC, and Marth JD. 1998. Immune regulation by the ST6Gal sialyltransferase. Proceedings of the National Academy of Sciences of the United States of America 95:4504-4509. 10.1073/pnas.95.8.4504

Jenkins N, Parekh RB, and James DC. 1996. Getting the glycosylation right: Implications for the biotechnology industry. Nature Biotechnology 14:975. 10.1038/nbt0896-975

Kavanaugh D, Kane M, Joshi L, and Hickey RM. 2013. Detection of galectin-3 interaction with commensal bacteria. Applied and Environmental Microbiology In press.

Kitazume-Kawaguchi S, Dohmae N, Takio K, Tsuji S, and Colley KJ. 1999. The relationship between ST6Gal I Golgi retention and its cleavage-secretion. Glycobiology 9:1397-1406.

Krzewinski-Recchi M-A, Julien S, Juliant S, Teintenier-Lelièvre M, Samyn-Petit B, Montiel M-D, Mir A-M, Cerutti M, Harduin-Lepers A, and Delannoy P. 2003. Identification and functional expression of a second human $\beta$-galactoside $\alpha 2,6$-sialyltransferase, ST6Gal II. European Journal of Biochemistry 270:950-961. 10.1046/j.1432-1033.2003.03458.x

Kudo T, Ikehara Y, Togayachi A, Morozumi K, Watanabe M, Nakamura M, Nishihara S, and Narimatsu H. 1998. Up-regulation of a set of glycosyltransferase genes in human colorectal cancer. Laboratory Investigation 78:797-811.

Kuhn B, Benz J, Greif M, Engel AM, Sobek H, and Rudolph MG. 2013. The structure of human alpha-2,6sialyltransferase reveals the binding mode of complex glycans. Acta Crystallographica Section DBiological Crystallography 69:1826-1838. 10.1107/s0907444913015412

Lane JA, Marino K, Naughton J, Kavanaugh D, Clyne M, Carrington SD, and Hickey RM. 2012. Antiinfective bovine colostrum oligosaccharides: Campylobacter jejuni as a case study. International Journal of Food Microbiology 157:182-188. 10.1016/j.ijfoodmicro.2012.04.027

Lee EU, Roth J, and Paulson JC. 1989. Alteration of terminal glycosylation sequences on N-linked oligosaccharides of Chinese hamster ovary cells by expression of beta-galactoside alpha 2,6sialyltransferase. Journal of Biological Chemistry 264:13848-13855. 
649

650

651

652

653

654

655

656

657

658

659

660

661

662

663

664

665

666

667

668

669

670

671

672

673

674

675

676

677

678

679

680

681

682

683

684

685

686

687

688

689

690

691

692

693

694

695

Lefébure R, Larsson S, and Byström P. 2011. A temperature-dependent growth model for the threespined stickleback Gasterosteus aculeatus. Journal Of Fish Biology 79:1815-1827.

10.1111/j.1095-8649.2011.03121.x

Legaigneur P, Breton C, El Battari A, Guillemot JC, Auge C, Malissard M, Berger EG, and Ronin C. 2001. Exploring the acceptor substrate recognition of the human beta-galactoside alpha 2,6sialyltransferase. Journal of Biological Chemistry 276:21608-21617. 10.1074/jbc.M100860200

Lehmann F, Kelm S, Dietz F, von Itzstein M, and Tiralongo J. 2008. The evolution of galactose alpha 2,3sialyltransferase: Ciona intestinalis ST3GAL I/II and Takifugu rubripes ST3GAL II sialylate Gal beta 1,3GalNAc structures on glycoproteins but not glycolipids. Glycoconjugate Journal 25:323-334. 10.1007/s10719-007-9078-4

Marino K, Lane JA, Abrahams JL, Struwe WB, Harvey DJ, Marotta M, Hickey RM, and Rudd PM. 2011. Method for milk oligosaccharide profiling by 2-aminobenzamide labeling and hydrophilic interaction chromatography. Glycobiology 21:1317-1330. 10.1093/glycob/cwr067

McCoy JP, Varani J, and Goldstein IJ. 1983. Enzyme-Linked Lectin Assay (ELLA) - Use of alkaline phosphatase-conjugated Griffonia simplicifolia B4 isolectin for the detection of alpha-Dgalactopyranosyl end groups. Analytical Biochemistry 130:437-444. Doi 10.1016/00032697(83)90613-9

McJarrow P, and van Amelsfort-Schoonbeek J. 2004. Bovine sialyl oligosaccharides: seasonal variations in their concentrations in milk, and a comparison of the colostrums of Jersey and Friesian cows. International Dairy Journal 14:571-579. 10.1016/j.idairyj.2003.11.006

Meng L, Forouhar F, Thieker D, Gao ZW, Ramiah A, Moniz H, Xiang Y, Seetharaman J, Milaninia S, Su M, Bridger R, Veillon L, Azadi P, Kornhaber G, Wells L, Montelione GT, Woods RJ, Tong L, and Moremen KW. 2013. Enzymatic Basis for N-Glycan Sialylation: Structure of rat alpha 2,6sialyltransferase (ST6Gal1) reveals conserved and unique features for glycan sialylation. Journal of Biological Chemistry 288:34680-34698. 10.1074/jbc.M113.519041

Monaco L, Marc A, Eon-Duval A, Acerbis G, Distefano G, Lamotte D, Engasser J-M, Soria M, and Jenkins N. 1996. Genetic engineering of $\alpha 2,6$-sialyltransferase in recombinant $\mathrm{CHO}$ cells and its effects on the sialylation of recombinant interferon- $\gamma$. Cytotechnology 22:197-203. 10.1007/bf00353939

Moremen KW, Ramiah A, Stuart M, Steel J, Meng L, Forouhar F, Moniz HA, Gahlay G, Gao ZW, Chapla D, Wang S, Yang JY, Prabhakar PK, Johnson R, dela Rosa M, Geisler C, Nairn AV, Seetharaman J, Wu SC, Tong L, Gilbert HJ, LaBaer J, and Jarvis DL. 2018. Expression system for structural and functional studies of human glycosylation enzymes. Nature Chemical Biology 14:156-+. 10.1038/nchembio.2539

Mysore JV, Wigginton T, Simon PM, Zopf D, Heman-Ackah LM, and Dubois A. 1999. Treatment of Helicobacter pylori infection in rhesus monkeys using a novel antiadhesion compound. Gastroenterology 117:1316-1325. 10.1016/s0016-5085(99)70282-9

Nakajima H, Nitta A, and Fujita K. 2010. Seasonal swimming behavior and optimum water temperature for adult ocellate puffer Takifugu rubripes revealed by using archival tags. Aquaculture Science 58:85-96. 10.11233/aquaculturesci.58.85

Olufsen M, Brandsdal BO, and Smalas AO. 2007. Comparative unfolding studies of psychrophilic and mesophilic uracil DNA glycosylase: MD simulations show reduced thermal stability of the coldadapted enzyme. Journal of Molecular Graphics \& Modelling 26:124-134. 10.1016/j.jmgm.2006.10.003

Onitsuka M, Kim W-D, Ozaki H, Kawaguchi A, Honda K, Kajiura H, Fujiyama K, Asano R, Kumagai I, Ohtake $H$, and Omasa T. 2012. Enhancement of sialylation on humanized IgG-like bispecific antibody by overexpression of $\alpha 2,6$-sialyltransferase derived from Chinese hamster ovary cells. Applied Microbiology and Biotechnology 94:69-80. 10.1007/s00253-011-3814-1 
696

697

698

699

700

701

702

703

704

705

706

707

708

709

710

711

712

713

714

715

716

717

718

719

720

721

722

723

724

725

726

727

728

729

730

731

732

733

734

735

736

737

738

739

740

741

742

743

Ortiz-Soto ME, and Seibel J. 2016. Expression of Functional Human Sialyltransferases ST3Gal1 and ST6Gal1 in Escherichia coli. Plos One 11. 10.1371/journal.pone.0155410

Petit D, Teppa E, Cenci U, Ball S, and Harduin-Lepers A. 2018. Reconstruction of the sialylation pathway in the ancestor of eukaryotes. Sci Rep 8. 10.1038/s41598-018-20920-1

Petit D, Teppa E, Mir AM, Vicogne D, Thisse C, Thisse B, Filloux C, and Harduin-Lepers A. 2015. Integrative view of alpha 2,3-sialyltransferases (ST3Gal) molecular and functional evolution in deuterostomes: significance of lineage-specific losses. Mol Biol Evol 32:906-927. 10.1093/molbev/msu395

Rao FV, Rich JR, Rakić B, Buddai S, Schwartz MF, Johnson K, Bowe C, Wakarchuk WW, DeFrees S, Withers SG, and Strynadka NCJ. 2009. Structural insight into mammalian sialyltransferases. Nature Structural \& Molecular Biology 16:1186-1188. 10.1038/nsmb.1685

Ribitsch D, Zitzenbacher S, Augustin P, Schmolzer K, Czabany T, Luley-Goedl C, Thomann M, Jung C, Sobek H, Muller R, Nidetzky B, and Schwab H. 2014. High-quality production of human alpha2,6-sialyltransferase in Pichia pastoris requires control over $\mathrm{N}$-terminal truncations by hostinherent protease activities. Microbial Cell Factories 13. 10.1186/s12934-014-0138-8

Sherry ST, Ward MH, Kholodov M, Baker J, Phan L, Smigielski EM, and Sirotkin K. 2001. dbSNP: the NCBI database of genetic variation. Nucleic Acids Research 29:308-311. 10.1093/nar/29.1.308

Siddiqui KS, and Cavicchioli R. 2006. Cold-adapted enzymes. Annual Review of Biochemistry. Palo Alto: Annual Reviews, 403-433.

Smith DF, Song X, and Cummings RD. 2010. Chapter 19 - Use of glycan microarrays to explore specificity of glycan-binding proteins. In: Fukuda M, ed. Methods in Enzymology: Academic Press, 417-444.

Swindall AF, Londono-Joshi Al, Schultz MJ, Fineberg N, Buchsbaum DJ, and Bellis SL. 2013. ST6Gal-I protein expression is upregulated in human epithelial tumors and correlates with stem cell markers in normal tissues and colon cancer cell lines. Cancer Res 73:2368-2378. 10.1158/00085472.CAN-12-3424

Takashima S, Tsuji S, and Tsujimoto M. 2002. Characterization of the second type of human betagalactoside alpha 2,6-sialyltransferase (ST6Gal II), which sialylates Galbeta 1,4GIcNAc structures on oligosaccharides preferentially. Genomic analysis of human sialyltransferase genes. J Biol Chem 277:45719-45728. 10.1074/jbc.M206808200

Teppa RE, Petit D, Plechakova O, Cogez V, and Harduin-Lepers A. 2016. Phylogenetic-derived insights into the evolution of sialylation in eukaryotes: Comprehensive analysis of vertebrate betagalactoside alpha 2,3/6-sialyltransferases (ST3Gal and ST6Gal). Int J Mol Sci 17:20. 10.3390/ijms17081286

Thompson R, Creavin A, O'Connell M, O'Connor B, and Clarke P. 2011. Optimization of the enzymelinked lectin assay for enhanced glycoprotein and glycoconjugate analysis. Analytical Biochemistry 413:114-122. 10.1016/j.ab.2011.02.013

Vallejo-Ruiz V, Haque R, Mir AM, Schwientek T, Mandel U, Cacan R, Delannoy P, and Harduin-Lepers A. 2001. Delineation of the minimal catalytic domain of human Gal beta 1-3GalNAc alpha 2,3sialyltransferase (hST3Gal I). Biochimica Et Biophysica Acta-Protein Structure and Molecular Enzymology 1549:161-173. 10.1016/s0167-4838(01)00257-6

Varki A. 1999. Essentials of Glycobiology: Cold Spring Harbor Laboratory Press \{a\}, 10 Skyline Drive, Plainview, New York 11803, USA.

Varki A. 2007. Glycan-based interactions involving vertebrate sialic-acid-recognizing proteins. Nature 446:1023-1029. 10.1038/nature05816

Volkers G, Worrall L, Kwan DH, Yu CC, Baumann L, Lameignere E, Wasney GA, Scott NE, Wakarchuk W, Foster LJ, Withers SG, and Strynadka NCJ. 2015. Structure of human ST8Sialll sialyltransferase provides insight into cell-surface polysialylation. Nature Structural \& Molecular Biology 22:627635. 10.1038/nsmb.3060

PeerJ reviewing PDF | (2018:05:28444:1:1:NEW 28 Aug 2018) 
744 Wu ZL, Ethen CM, Prather B, Machacek M, and Jiang WP. 2011. Universal phosphatase-coupled

745 glycosyltransferase assay. Glycobiology 21:727-733. 10.1093/glycob/cwq187

746 Yamamoto T, Takakura Y, and Tsukamoto H. 2006. Bacterial sialyltransferases. Trends in Glycoscience

$747 \quad$ and Glycotechnology 18:253-265. 10.4052/tigg.18.253

748 


\section{Figure 1}

Sialyltransferase fusion construct detail

The sialyltransferase constructs (ST6Gal I, ST6Gal II and ST3Gal IV) all have this general structure. The specifics relate to human ST6Gal I. An Igk leader sequence is fused in frame with an attB1 sequence (consequence of Gateway recombination), the SIAT sequence, an attB2 sequence and the $\mathrm{V} 5-\mathrm{His}_{6} \mathrm{C}$-terminal tag. $\mathrm{L}$ and $\mathrm{S}$ motifs, common to all SIATs are indicated. Cysteine residues (human ST6Gal I) are paired 142/406 (red arrows), 184/335 (blue) and 353/364 (green). Cys-184 is the reference point chosen to select the start of the SIAT catalytic domain: for ST6Gal I proteins this was 121 amino acids (aa) towards the Nterminal of the protein and for ST3Gal IV proteins this was 70 amino acids (aa) towards the $\mathrm{N}$-terminal of the protein. 


$$
\begin{aligned}
& x=121 \text { aa for } S T 6 G a l \\
& x=70 \text { aa for ST3Gal }
\end{aligned}
$$

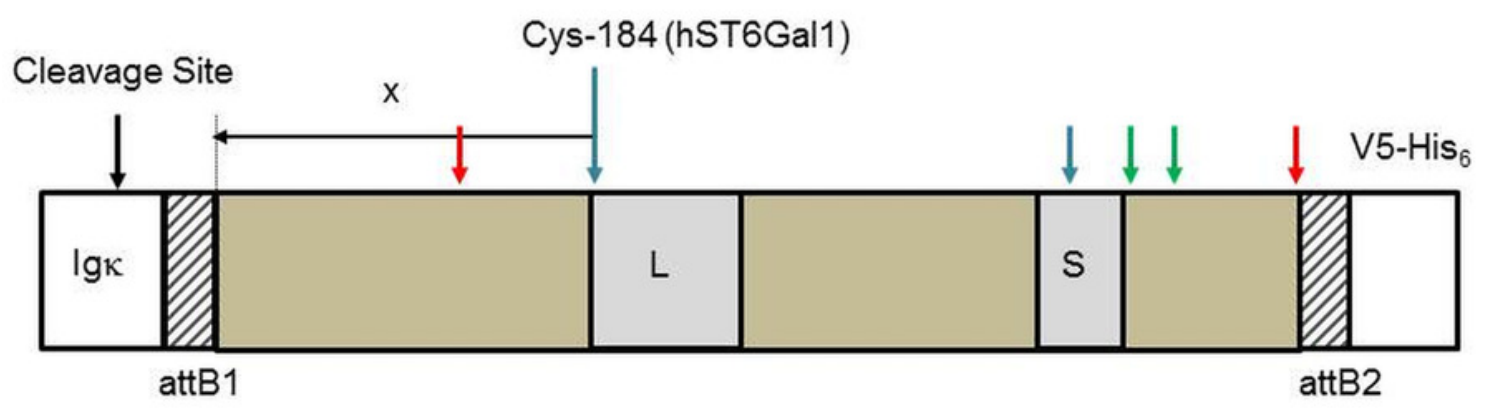




\section{Figure 2}

Lectin binding to $\mathrm{CHO}$ cells expressing ST6Gal I

CHO cells expressing ST6Gal I show strong binding of SNA-I (A) and moderate binding of MAA (B). CHO cells (non-transfected control) showed weak binding of SNA-I (C) and moderate binding of MAA (D). Lectins were FITC-labelled and cells were counterstained with DAPI. Binding of SNA-I was inhibited by the presence of 100 mM lactose (Figure S3)

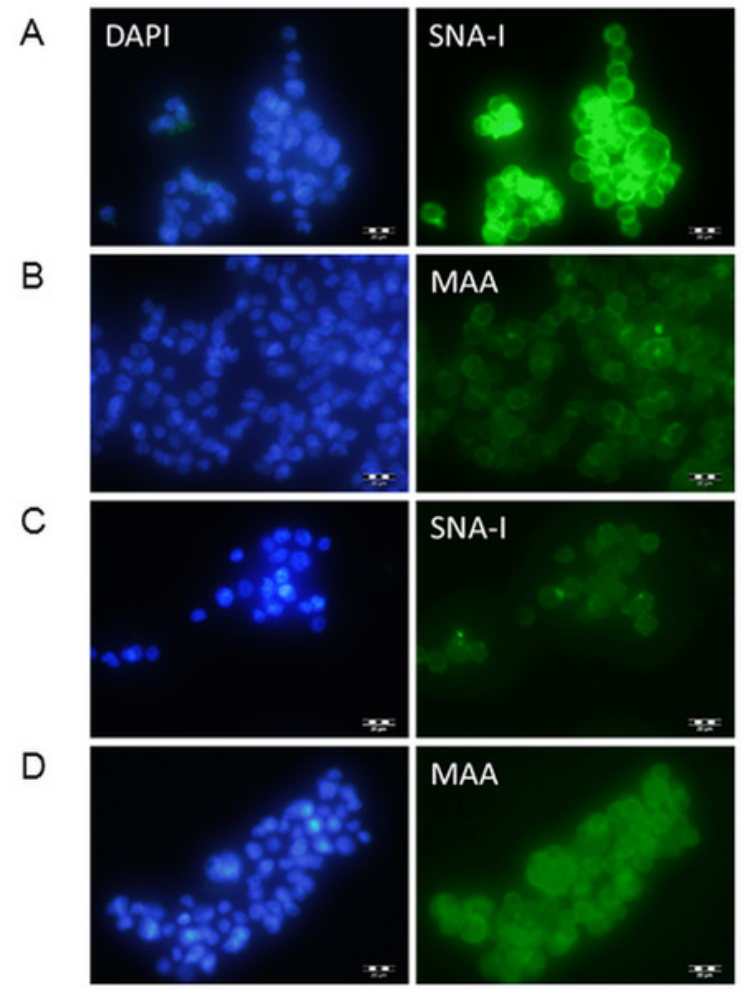




\section{Figure 3}

Sialyltransferase assay on purified enzyme preparations

Proteins purified on Ni Sepharose excel (150 ng) were assayed using LacNAc (2.4 mM) as acceptor and CMP-Neu5Ac (0.2 mM) as donor. Reactions were incubated for 30 min at $37{ }^{\circ} \mathrm{C}$. Transfer of Neu5Ac was measured by release of phosphate using a phosphate linked assay with Malachite Green detection. These are the averages of three separate experiments. Human ST6Gal II is shown with lighter shading and the negative control (Neg) (IMAC-purified PSA) has no shading. Mouse ST6Gal I (mST6) is a commercial preparation used as a positive control. See Table 1 for explanation of all clone names. Significant activities following Student $t$-tests between PSA control and SIAT clones are marked with an asterisk $(p<0.01)$.

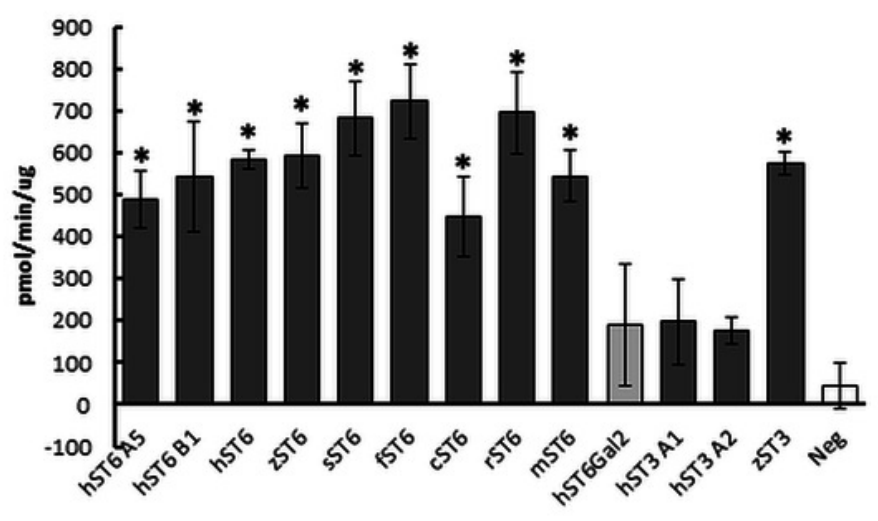




\section{Figure 4}

Western blots of purified proteins

A. Various ST6Gal I and ST3Gal IV purified proteins on Western blots detected by mouse antiHis, HRP-goat anti-mouse IgG with DAB staining. B. PNGase F digested ST6Gal I (+) and undigested (-) control. Loading was 2ug purified from ProCHO-AT media separated on $12 \%$ NuPAGE Novex Bis-Tris gels in MOPS buffer under reducing conditions. Marker: BenchMark His-tagged protein standard. See Table 1 for explanation of all clone names.

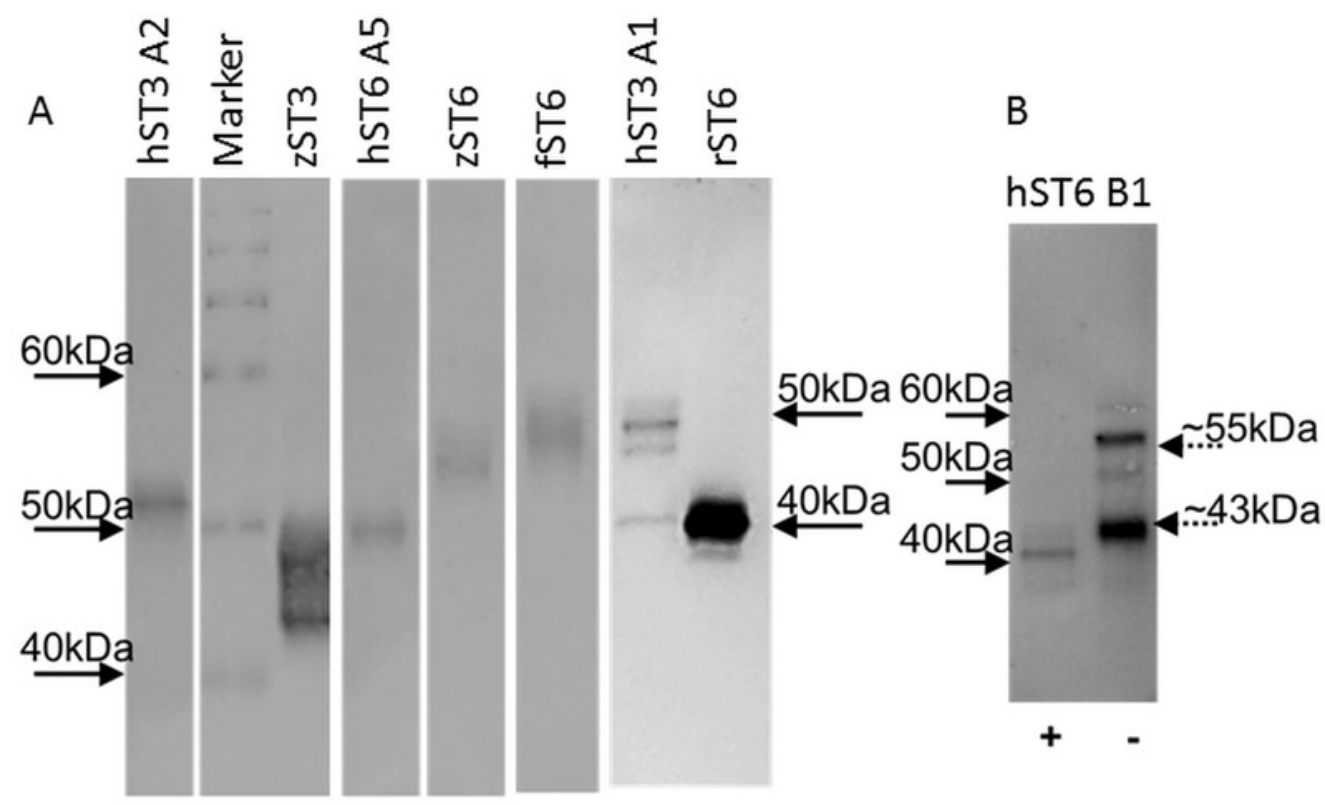


Figure 5

HILIC chromatographs for typical sialyltransferase incubations

A. Overlay of 2-AB labelled whey permeate ( $80 \%$ lactose), $3^{\prime}$ sialyllactose (3'SL) and 6' sialyllactose (6'SL) standards. B-E. 2-AB labelled glycans after incubation of whey permeate with various sialyltransferases. Reaction conditions were $16 \mathrm{~h}$ at $37^{\circ} \mathrm{C}$ (or $20^{\circ} \mathrm{C}$ ) at $8 \mathrm{mM}$ lactose (in whey permeate) and 2 mM CMP-Neu5Ac. B. Human ST6Gal I clone B1 (hST6 B1) at $20^{\circ} \mathrm{C}$. C. Commercial ST6Gal I (mST6) (Sino Biologicals). D. Stickleback ST6Gal I (sST6). E. Human ST3Gal IV clone A1 (hST3 A1). Peaks have consistent and diagnostic GU (glucose unit) values calibrated to a dextran standard across runs.

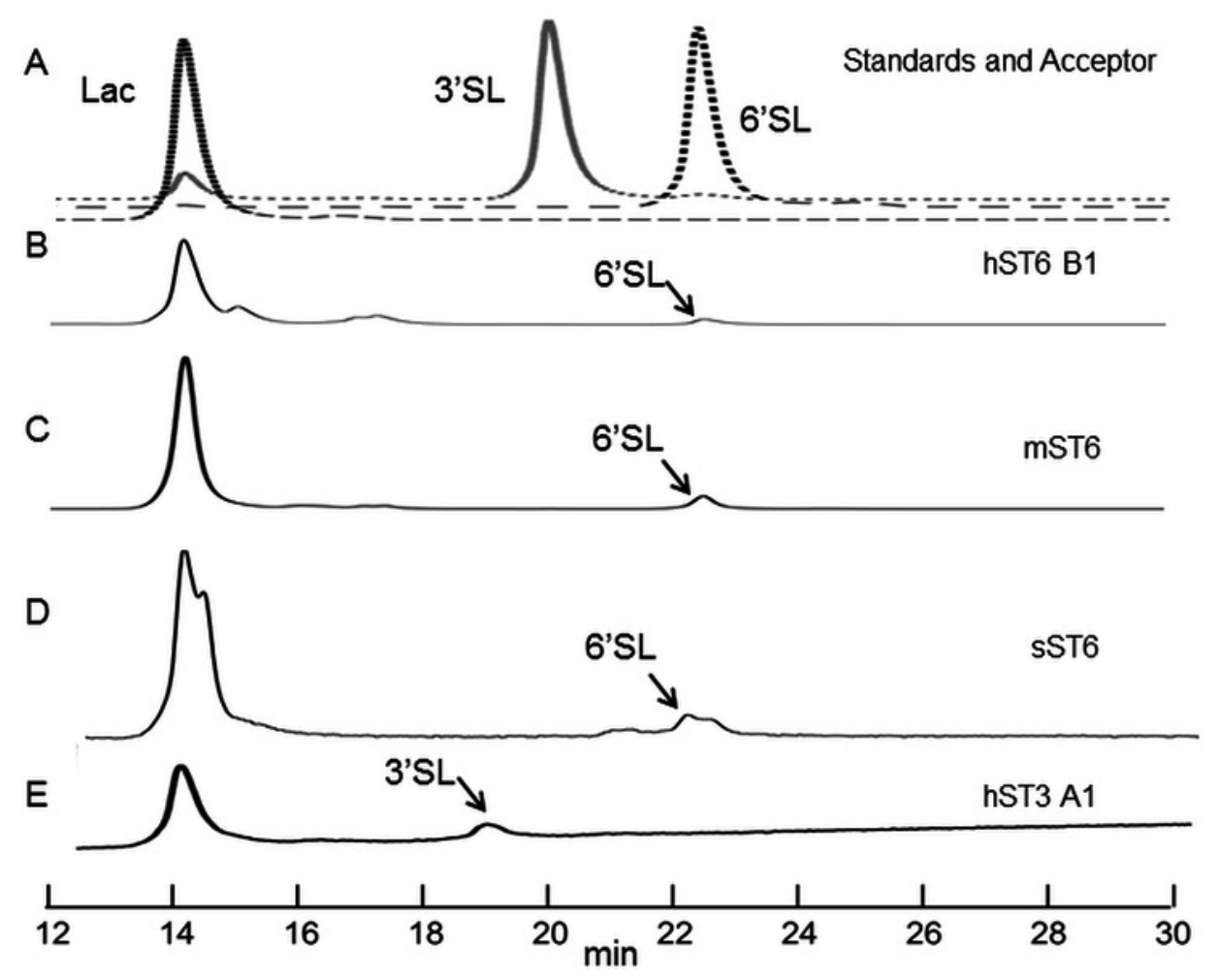




\section{Figure 6}

Enzyme-linked lectin assay

A. ASF, reacted with each of the sialyltransferase preparations $\left(37^{\circ} \mathrm{C}, 16 \mathrm{~h}\right.$ ), was bound to biotinylated SNA-I lectin. Binding to Neu5Ac on the ASF was detected with HRP-streptavidin. Expt 1 (black bars) included ST6Gal I, ST3Gal IV and ST6Gal II preparations. Expt 2 (grey bars) included only ST6Gal I preparations. B. Each sample was analysed for binding with biotinylated RCA I lectin. All values are relative to ASF. RCA I will bind strongly to Gal residues on ASF but binding will decrease as Gal residues are sialylated with each of the ST6Gal I, ST6Gal II and ST3Gal IV sialyltransferases. Each sample was assayed in triplicate and error bars show SD. FET, fetuin (positive control); ASF, asialofetuin. Significant binding events following Student $t$-tests are marked with an asterisk $(p<0.01)$. 


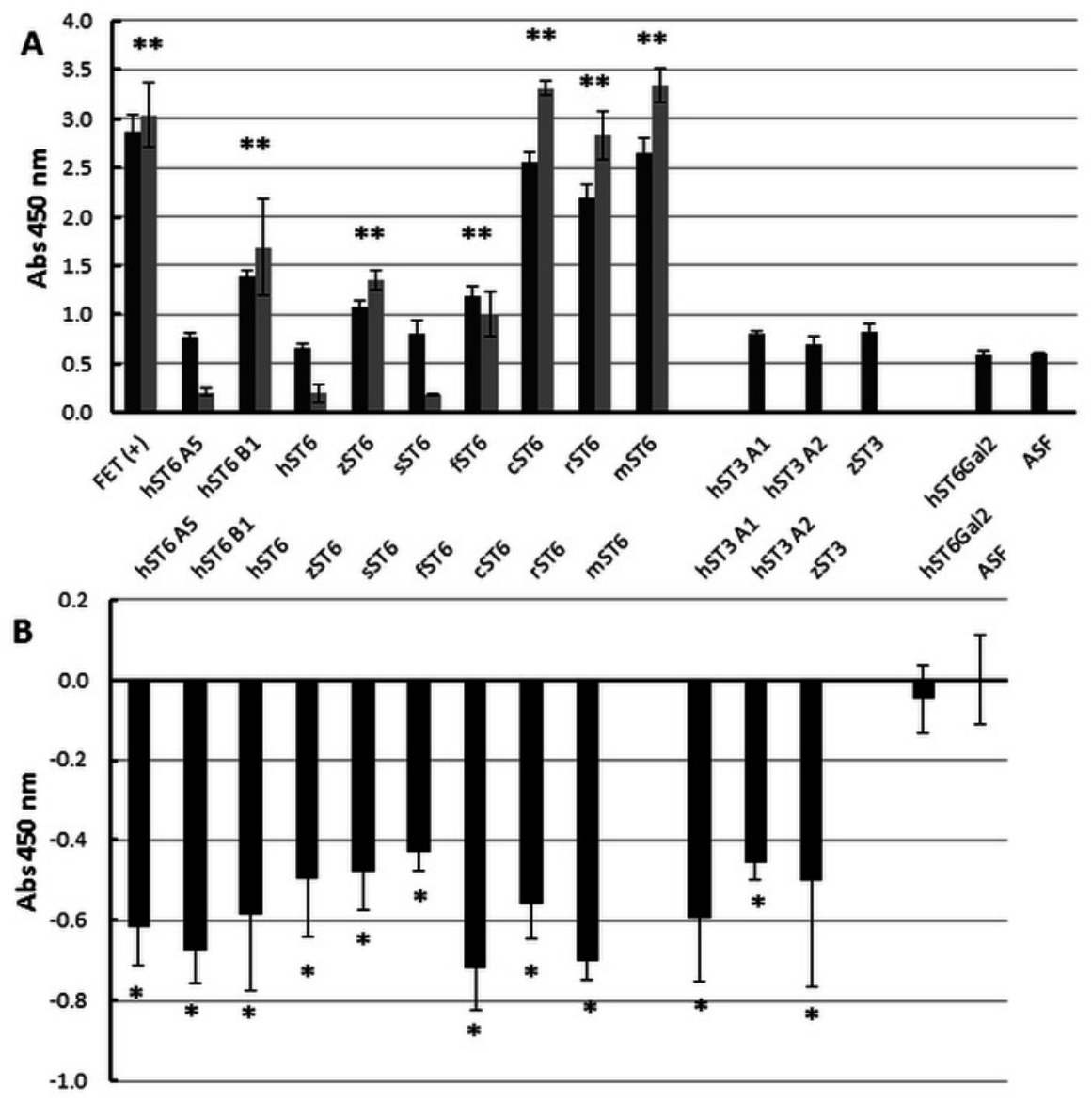




\section{Table $\mathbf{1}$ (on next page)}

Summary of all constructs

All fusion proteins start with the Igk leader sequence followed by the Gateway linkers and the catalytic domain of the sialyltransferase (SIAT). 
1 Table 1:

2

3

4

\begin{tabular}{llllll}
\hline Protein & Clone & Species & Accession No. & $\begin{array}{l}\text { Catalytic } \\
\text { start }\end{array}$ & SIAT (first 15 residues) \\
\hline ST6Gal I & hST6 & Human & NP_003023.1 & Val-63 & VSSSSTQDPHRGRQT \\
ST6Gal I & hST6 A5 & Human & NP_003023.1 & Val-63 & VSSSSTQDPHRGRQT \\
ST6Gal I & hST6 B1 & Human & NP_003023.1 & Val-63 & VSSSSTQDPHRGRQT \\
ST6Gal I & zST6 & Zebrafish & NP_001003853.1 & Val-63 & VKVLRGTGGSKPMYT \\
ST6Gal I & rST6 & Rat & P13721.1 & Val-60 & VFSNSKQDPKEDIPI \\
ST6Gal I & sST6 & Stickleback & CBQ74103.1 & Thr-136 & TLFGGRRRGELSGRV \\
ST6Gal I & fST6 & Fugu & NP_001027933.1 & Thr-136 & TLFGGRRKGELSGRG \\
ST6Gal I & cST6 & Chicken & XP_015132322 & Gln-69 & QMPKALPNNQNKVKG \\
ST6Gal II & hST6Gal2 & Human & NP_115917 & Val-173 & VKKRHRRQRRSHVLE \\
ST3Gal IV & hST3 A1 & Human & AF525084_1 & Ala-47 & AESKASKLFGKLSPL \\
ST3Gal IV & hST3 A2 & Human & NP_001241686 & Ala-47 & AESKASKLFGNYSRD \\
ST3Gal IV & z ST3 H6 & Zebrafish & NP_001076498 & Glu-55 & ENLNLNMSRKPELFL \\
\hline A1l fusion & proteins & & Wtart the &
\end{tabular}

5 All fusion proteins start with the Igא leader sequence followed by the Gateway linkers and the

6 catalytic domain of the sialyltransferase (SIAT). 